\title{
Sharp Bounds by the Generalized Logarithmic Mean for the Geometric Weighted Mean of the Geometric and Harmonic Means
}

\author{
Wei-Mao Qian' ${ }^{1}$ and Bo-Yong Long ${ }^{2}$ \\ ${ }^{1}$ School of Distance Education, Huzhou Broadcast and TV University, Huzhou 313000, China \\ ${ }^{2}$ School of Mathematics Science, Anhui University, Hefei 230039, China
}

Correspondence should be addressed to Wei-Mao Qian, qwm661977@126.com

Received 29 January 2012; Revised 19 February 2012; Accepted 12 March 2012

Academic Editor: Yuri Sotskov

Copyright (C) 2012 W.-M. Qian and B.-Y. Long. This is an open access article distributed under the Creative Commons Attribution License, which permits unrestricted use, distribution, and reproduction in any medium, provided the original work is properly cited.

We present sharp upper and lower generalized logarithmic mean bounds for the geometric weighted mean of the geometric and harmonic means.

\section{Introduction}

For $p \in \mathbb{R}$ the generalized logarithmic mean $L_{p}(a, b)$ of two positive numbers $a$ and $b$ is defined by

$$
L_{p}(a, b)= \begin{cases}a, & a=b, \\ {\left[\frac{a^{p+1}-b^{p+1}}{(p+1)(a-b)}\right]^{1 / p},} & p \neq 0, p \neq-1, a \neq b, \\ \frac{1}{e}\left(\frac{b^{b}}{a^{a}}\right)^{1 /(b-a)}, & p=0, a \neq b, \\ \frac{b-a}{\log b-\log a}, & p=-1, a \neq b .\end{cases}
$$

It is well-known that $L_{p}(a, b)$ is continuous and strictly increasing with respect to $p \in \mathbb{R}$ for fixed $a$ and $b$ with $a \neq b$. In the recent past, the generalized logarithmic mean has been the subject of intensive research. In particular, many remarkable inequalities for $L_{p}$ can be 
found in the literature [1-23]. The generalized logarithmic mean has applications in convex function, economics, physics, and even in meteorology [24-27]. In [26] the authors study a variant of Jensen's functional equation involving $L_{p}$, which appear in a heat conduction problem. Let $A(a, b)=(a+b) / 2, I(a, b)=(1 / e)\left(b^{b} / a^{a}\right)^{1 /(b-a)}, L(a, b)=(b-a) /(\log b-\log a)$, $G(a, b)=\sqrt{a b}$, and $H(a, b)=2 a b /(a+b)$ be the arithmetic, identric, logarithmic, geometric, and harmonic means of two positive numbers $a$ and $b$ with $a \neq b$, respectively. Then it is well known that

$$
\begin{aligned}
\min \{a, b\} & <H(a, b)<G(a, b)=L_{-2}(a, b)<L(a, b)=L_{-1}(a, b) \\
& <I(a, b)=L_{0}(a, b)<A(a, b)=L_{1}(a, b)<\max \{a, b\} .
\end{aligned}
$$

In [28-30], the authors present bounds for $L$ and $I$ in terms of $G$ and $A$.

Proposition 1.1. For all positive real numbers $a$ and $b$ with $a \neq b$, one has

$$
\begin{gathered}
A^{1 / 3}(a, b) G^{2 / 3}(a, b)<L(a, b)<\frac{1}{3} A(a, b)+\frac{2}{3} G(a, b), \\
\frac{1}{3} G(a, b)+\frac{2}{3} A(a, b)<I(a, b) .
\end{gathered}
$$

The proof of the following Proposition 1.2 can be found in [31].

Proposition 1.2. For all positive real numbers $a$ and $b$ with $a \neq b$, we have

$$
\sqrt{G(a, b) A(a, b)}<\sqrt{L(a, b) I(a, b)}<\frac{1}{2}(L(a, b)+I(a, b))<\frac{1}{2}(G(a, b)+A(a, b)) .
$$

For $r \in \mathbb{R}$ the $r$ th power mean $M_{r}(a, b)$ of two positive numbers $a$ and $b$ is defined by

$$
M_{r}(a, b)= \begin{cases}\left(\frac{a^{r}+b^{r}}{2}\right)^{1 / r}, & r \neq 0 \\ \sqrt{a b}, & r=0 .\end{cases}
$$

The main properties of these means are given in [32]. Several authors discussed the relationship of certain means to $M_{r}$. The following sharp bounds for $L, I,(I L)^{1 / 2}$, and $(I+$ $L) / 2$ in terms of power means are proved in [31,33-37].

Proposition 1.3. For all positive real numbers $a$ and $b$ with $a \neq b$ one has

$$
\begin{gathered}
M_{0}(a, b)<L(a, b)<M_{1 / 3}(a, b), \quad M_{2 / 3}(a, b)<I(a, b)<M_{\log 2}(a, b), \\
M_{0}(a, b)<I^{1 / 2}(a, b) L^{1 / 2}(a, b)<M_{1 / 2}(a, b), \\
\frac{1}{2}[I(a, b)+L(a, b)]<M_{1 / 2}(a, b) .
\end{gathered}
$$

The following three results were established by Alzer and Qiu in [38]. 
Proposition 1.4. The inequalities

$$
\alpha A(a, b)+(1-\alpha) G(a, b)<I(a, b)<\beta A(a, b)+(1-\beta) G(a, b)
$$

hold for all positive real numbers $a$ and $b$ with $a \neq b$ if and only if

$$
\alpha \leq \frac{2}{3}, \quad \beta \geq \frac{2}{e}=0.73575 \cdots
$$

Proposition 1.5. Let $a$ and $b$ be real numbers with $a \neq b$. If $0<a, b \leq e$, then

$$
[G(a, b)]^{A(a, b)}<[L(a, b)]^{I(a, b)}<[A(a, b)]^{G(a, b)} .
$$

And, if $a, b \geq e$, then

$$
[A(a, b)]^{G(a, b)}<[I(a, b)]^{L(a, b)}<[G(a, b)]^{A(a, b)} .
$$

Proposition 1.6. For all positive real numbers $a$ and $b$ with $a \neq b$, one has

$$
M_{c}(a, b)<\frac{1}{2}(L(a, b)+I(a, b))
$$

with the best possible parameter $c=\log 2 /(1+\log 2)=0.40938 \cdots$

In [39] the authors presented inequalities between the generalized logarithmic mean and the product $A^{\alpha}(a, b) G^{\beta}(a, b) H^{\gamma}(a, b)$ for all $a, b>0$ with $a \neq b$ and $\alpha, \beta>0$ with $\alpha+\beta<1$.

It is the aim of this paper to give a solution to the problem: for $\alpha \in(0,1)$, what are the greatest value $p$ and the least value $q$, such that the inequality

$$
L_{p}(a, b) \leq G^{\alpha}(a, b) H^{1-\alpha}(a, b) \leq L_{q}(a, b)
$$

holds for all $a, b>0$ ?

\section{Main Result}

Theorem 2.1. For $\alpha \in(0,1)$ and all $a, b>0$, one has the following:

(1) $L_{3 \alpha-5}(a, b)=G^{\alpha}(a, b) H^{1-\alpha}(a, b)=L_{-(2 / \alpha)}(a, b)$ for $\alpha=2 / 3$,

(2) $L_{3 \alpha-5}(a, b) \geq G^{\alpha}(a, b) H^{1-\alpha}(a, b) \geq L_{-(2 / \alpha)}(a, b)$ for $0<\alpha<2 / 3$, and $L_{3 \alpha-5}(a, b) \leq$ $G^{\alpha}(a, b) H^{1-\alpha}(a, b) \leq L_{-(2 / \alpha)}(a, b)$ for $2 / 3<\alpha<1$, with equality if and only if $a=b$, and the parameters $3 \alpha-5$ and $-2 / \alpha$ in each inequality cannot be improved.

Proof. (1) If $\alpha=2 / 3$ and $a=b$, then (1.1) implies that $L_{3 \alpha-5}(a, b)=G^{\alpha}(a, b) H^{1-\alpha}(a, b)=$ $L_{-(2 / \alpha)}(a, b)=a$. 
If $\alpha=2 / 3$ and $a \neq b$, then (1.1) leads to

$$
\begin{aligned}
L_{3 \alpha-5}(a, b) & =L_{-(2 / \alpha)}(a, b)=L_{-3}(a, b)=\left[\frac{a^{-2}-b^{-2}}{2(b-a)}\right]^{-1 / 3} \\
& =(a b)^{1 / 3}\left(\frac{2 a b}{a+b}\right)^{1 / 3}=G^{2 / 3}(a, b) H^{1 / 3}(a, b)=G^{\alpha}(a, b) H^{1-\alpha}(a, b) .
\end{aligned}
$$

(2) If $a=b$, then from (1.1) we clearly see that $L_{3 \alpha-5}(a, b)=G^{\alpha}(a, b) H^{1-\alpha}(a, b)=$ $L_{-(2 / \alpha)}(a, b)=a$ for any $\alpha \in(0,1)$.

If $a \neq b$, without loss of generality, we assume $a>b$. Let $a / b=t>1$ and

$$
f(t)=\log L_{3 \alpha-5}(a, b)-\log \left[G^{\alpha}(a, b) H^{1-\alpha}(a, b)\right] .
$$

Then (1.1) and simple computations yield

$$
\begin{gathered}
f(t)=\frac{1}{3 \alpha-5} \log \frac{t^{3 \alpha-4}-1}{(3 \alpha-4)(t-1)}-\frac{\alpha}{2} \log t-(1-\alpha) \log \frac{2 t}{1+t^{\prime}} \\
\lim _{t \rightarrow 1^{+}} f(t)=0 \\
f^{\prime}(t)=-\frac{t^{4-3 \alpha}}{t\left(t^{2}-1\right)\left(t^{4-3 \alpha}-1\right)} g(t),
\end{gathered}
$$

where $g(t)=(2-\alpha / 2) t^{3 \alpha-2}-((2-\alpha)(2-3 \alpha) / 5-3 \alpha) t^{3 \alpha-3}+((1-\alpha)(2-3 \alpha) / 2(5-3 \alpha)) t^{3 \alpha-4}-$ $((1-\alpha)(2-3 \alpha) / 2(5-3 \alpha)) t^{2}+((2-\alpha)(2-3 \alpha) /(5-3 \alpha)) t-(2-\alpha) / 2$,

$$
\begin{aligned}
g(1)= & 0 \\
g^{\prime}(t)= & \frac{(2-\alpha)(3 \alpha-2)}{2} t^{3 \alpha-3}-\frac{3(2-\alpha)(2-3 \alpha)(\alpha-1)}{5-3 \alpha} t^{3 \alpha-4} \\
& +\frac{(1-\alpha)(2-3 \alpha)(3 \alpha-4)}{2(5-3 \alpha)} t^{3 \alpha-5}-\frac{(1-\alpha)(2-3 \alpha)}{(5-3 \alpha)} t \\
& +\frac{(2-\alpha)(2-3 \alpha)}{(5-3 \alpha)} \\
g^{\prime}(1)= & 0, \\
g^{\prime \prime}(t)= & \frac{3(2-\alpha)(3 \alpha-2)(\alpha-1)}{2} t^{3 \alpha-4}-\frac{3(2-\alpha)(2-3 \alpha)(\alpha-1)(3 \alpha-4)}{5-3 \alpha} t^{3 \alpha-5} \\
& -\frac{(1-\alpha)(2-3 \alpha)(3 \alpha-4)}{2} t^{3 \alpha-6}-\frac{(1-\alpha)(2-3 \alpha)}{(5-3 \alpha)} \\
g^{\prime \prime}(1)= & 0, \\
g^{\prime \prime \prime}(t)= & \frac{3}{2}(1-\alpha)(2-\alpha)(4-3 \alpha)(3 \alpha-2) t^{3 \alpha-7}(t-1)^{2} .
\end{aligned}
$$


Journal of Applied Mathematics

If $0<\alpha<2 / 3$, then (2.7) implies

$$
g^{\prime \prime \prime}(t)<0
$$

for $t>1$.

From (2.3)-(2.6) and (2.8) we know that $f(t)>0$ for $t>1$.

If $2 / 3<\alpha<1$, then (2.7) leads to

$$
g^{\prime \prime \prime}(t)>0
$$

for $t>1$. Therefore $f(t)<0$ for $t>1$ follows from (2.3)-(2.6) and (2.9).

Let

$$
h(t)=\log L_{-(2 / \alpha)}(a, b)-\log \left[G^{\alpha}(a, b) H^{1-\alpha}(a, b)\right]
$$

for $t=a / b>1$; then (1.1) and elementary calculations lead to

$$
\begin{gathered}
h(t)=-\frac{\alpha}{2} \log \frac{t^{(\alpha-2) / \alpha}-1}{((\alpha-2) / \alpha)(t-1)}-\frac{\alpha}{2} \log t-(1-\alpha) \log \frac{2 t}{1+t^{\prime}} \\
\lim _{t \rightarrow 1^{+}} h(t)=0, \\
h^{\prime}(t)=-\frac{t^{(2-\alpha) / \alpha}}{t\left(t^{2}-1\right)\left(t^{(2-\alpha) / \alpha}-1\right)} v(t),
\end{gathered}
$$

where $v(t)=((2-\alpha) / 2) t^{(3 \alpha-2) / \alpha}+((3 \alpha-2) / 2) t^{(2 \alpha-2) / \alpha}-((3 \alpha-2) / 2) t-(2-\alpha) / 2$,

$$
\begin{aligned}
v(1) & =0 \\
v^{\prime}(t) & =\frac{(2-\alpha)(3 \alpha-2)}{2 \alpha} t^{(2 \alpha-2) / \alpha}+\frac{(3 \alpha-2)(\alpha-1)}{\alpha} t^{(\alpha-2) / \alpha}-\frac{3 \alpha-2}{2}, \\
v^{\prime}(1) & =0 \\
v^{\prime \prime}(t) & =\frac{(2-\alpha)(1-\alpha)(2-3 \alpha)}{\alpha^{2}} t^{-2 / \alpha}(t-1) .
\end{aligned}
$$

If $\alpha \in(0,2 / 3)$, then (2.15) implies

$$
v^{\prime \prime}(t)>0
$$

for $t>1$.

From (2.11)-(2.14) and (2.16) we know that $h(t)<0$ for $t>1$.

If $\alpha \in(2 / 3,1)$, then (2.15) leads to

$$
v^{\prime \prime}(t)<0
$$

for $t>1$. Therefore, $h(t)>0$ for $t>1$ follows from (2.11)-(2.14) and (2.17). 
Next, we prove that the parameters $-(2 / \alpha)$ and $3 \alpha-5$ in either case cannot be improved. The proof is divided into two cases.

Case $1(\alpha \in(0,2 / 3))$. For any $\epsilon>0$ and $x \in(0,1)$, from (1.1) one has

$$
\begin{gathered}
{\left[G^{\alpha}(1,1+x) H^{1-\alpha}(1,1+x)\right]^{5-3 \alpha+\epsilon}-\left[L_{3 \alpha-5-\epsilon}(1,1+x)\right]^{5-3 \alpha+\epsilon}} \\
\quad=\frac{f_{1}(x)}{(1+x / 2)^{(1-\alpha)(5-3 \alpha+\epsilon)}\left[(1+x)^{4-3 \alpha+\epsilon}-1\right]}
\end{gathered}
$$

where $f_{1}(x)=(1+x)^{(1-\alpha / 2)(5-3 \alpha+\epsilon)}\left[(1+x)^{4-3 \alpha+\epsilon}-1\right]-(4-3 \alpha+\epsilon) x(1+x)^{4-3 \alpha+\epsilon}$ $(1+x / 2)^{(1-\alpha)(5-3 \alpha+\varepsilon)}$.

Let $x \rightarrow 0$; making use of the Taylor expansion, we get

$$
f_{1}(x)=\frac{\epsilon(4-3 \alpha+\epsilon)(5-3 \alpha+\epsilon)}{24} x^{3}+o\left(x^{3}\right) .
$$

Equations (2.18) and (2.19) imply that for any $\alpha \in(0,2 / 3)$ and $\epsilon>0$ there exists $\delta=\delta(\epsilon, \alpha) \in(0,1)$, such that $L_{3 \alpha-5-\epsilon}(1,1+x)<G^{\alpha}(1,1+x) H^{1-\alpha}(1,1+x)$ for $x \in(0, \delta)$.

On the other hand, for any $\epsilon \in(0,(2 / \alpha)-1)$ we have

$$
\begin{aligned}
& L_{-(2 / \alpha)+\epsilon}(1, t)-G^{\alpha}(1, t) H^{1-\alpha}(1, t) \\
& \quad=t^{\alpha /(2-\epsilon \alpha)}\left\{\left[\frac{1-t^{-2 / \alpha+\epsilon+1}}{(2 / \alpha-\epsilon-1)(1-1 / t)}\right]^{-\alpha /(2-\epsilon \alpha)}-t^{-\epsilon \alpha^{2} / 2(2-\epsilon \alpha)}\left(\frac{2 t}{1+t}\right)^{1-\alpha}\right\}, \\
& \lim _{t \rightarrow+\infty}\left\{\left[\frac{1-t^{-2 / \alpha+\epsilon+1}}{(2 / \alpha-\epsilon-1)(1-1 / t)}\right]^{-\alpha /(2-\epsilon \alpha)}-t^{-\epsilon \alpha^{2} / 2(2-\epsilon \alpha)}\left(\frac{2 t}{1+t}\right)^{1-\alpha}\right\} \\
& \quad=\left(\frac{2}{\alpha}-\epsilon-1\right)^{\alpha /(2-\epsilon \alpha)}>0 .
\end{aligned}
$$

From (2.20) we know that for any $\alpha \in(0,2 / 3)$ and $\epsilon \in(0,2 / \alpha-1)$ there exists $T=$ $T(\epsilon, \alpha)>1$, such that $L_{-2 / \alpha+\epsilon}(1, t)>G^{\alpha}(1, t) H^{1-\alpha}(1, t)$ for $t \in(T, \infty)$.

Case $2(\alpha \in(2 / 3,1))$. For any $\epsilon \in(0,4-3 \alpha)$ and $x \in(0,1)$, from (1.1) one has

$$
\begin{gathered}
{\left[L_{3 \alpha-5+\epsilon}(1,1+x)\right]^{5-3 \alpha-\epsilon}-\left[G^{\alpha}(1,1+x) H^{1-\alpha}(1,1+x)\right]^{5-3 \alpha-\epsilon}} \\
=\frac{f_{2}(x)}{(1+x / 2)^{(1-\alpha)(5-3 \alpha-\epsilon)}\left[(1+x)^{4-3 \alpha-\epsilon}-1\right]}
\end{gathered}
$$

where $f_{2}(x)=(4-3 \alpha-\epsilon) x(1+x)^{4-3 \alpha-\epsilon}(1+x / 2)^{(1-\alpha)(5-3 \alpha-\epsilon)}-(1+x)^{(1-\alpha / 2)(5-3 \alpha-\epsilon)}\left[(1+x)^{4-3 \alpha-\epsilon}-1\right]$.

Let $x \rightarrow 0$; making use of the Taylor expansion, we have

$$
f_{2}(x)=\frac{\epsilon}{24}(4-3 \alpha-\epsilon)(5-3 \alpha-\epsilon) x^{3}+o\left(x^{3}\right) .
$$


Equations (2.21) and (2.22) imply that for any $\alpha \in(2 / 3,1)$ and $\epsilon \in(0,4-3 \alpha)$ there exists $\delta=\delta(\epsilon, \alpha) \in(0,1)$, such that $L_{3 \alpha-5+\epsilon}(1,1+x)>G^{\alpha}(1,1+x) H^{1-\alpha}(1,1+x)$ for $x \in(0, \delta)$.

On the other hand, for any $\epsilon>0$, we have

$$
\begin{aligned}
& G^{\alpha}(1, t) H^{1-\alpha}(1, t)-L_{-(2 / \alpha)-\epsilon}(1, t) \\
& \quad=t^{\alpha / 2}\left\{\left(\frac{2 t}{1+t}\right)^{1-\alpha}-t^{-\epsilon \alpha^{2} / 2(2+\epsilon \alpha)}\left[\frac{1-t^{-(2 / \alpha+\epsilon-1)}}{(2 / \alpha+\epsilon-1)(1-1 / t)}\right]^{-\alpha /(2+\epsilon \alpha)}\right\}, \\
& \lim _{t \rightarrow+\infty}\left\{\left(\frac{2 t}{1+t}\right)^{1-\alpha}-t^{-\epsilon \alpha^{2} / 2(2+\epsilon \alpha)}\left[\frac{1-t^{-(2 / \alpha+\epsilon-1)}}{(2 / \alpha+\epsilon-1)(1-1 / t)}\right]^{-\alpha /(2+\epsilon \alpha)}\right\}=2^{1-\alpha}>0 .
\end{aligned}
$$

From (2.23) we know that for any $\alpha \in(2 / 3,1)$ and $\epsilon>0$ there exists $T=T(\epsilon, \alpha)>1$, such that $L_{-(2 / \alpha)-\epsilon}(1, t)<G^{\alpha}(1, t) H^{1-\alpha}(1, t)$ for $t \in(T, \infty)$.

\section{Acknowledgment}

This work was supported by the Natural Science Foundation of Zhejiang Broad-cast and TV University under Grant XKT-09G21.

\section{References}

[1] K. B. Stolarsky, "The power and generalized logarithmic means," The American Mathematical Monthly, vol. 87, no. 7, pp. 545-548, 1980.

[2] F. Qi and B.-N. Guo, "An inequality between ratio of the extended logarithmic means and ratio of the exponential means," Taiwanese Journal of Mathematics, vol. 7, no. 2, pp. 229-237, 2003.

[3] C.-P. Chen and F. Qi, "Monotonicity properties for generalized logarithmic means," The Australian Journal of Mathematical Analysis and Applications, vol. 1, no. 2, article 2, p. 4, 2004.

[4] X. Li, C.-P. Chen, and F. Qi, "Monotonicity result for generalized logarithmic means," Tamkang Journal of Mathematics, vol. 38, no. 2, pp. 177-181, 2007.

[5] F. Qi, S.-X. Chen, and C.-P. Chen, "Monotonicity of ratio between the generalized logarithmic means," Mathematical Inequalities E Applications, vol. 10, no. 3, pp. 559-564, 2007.

[6] C.-P. Chen, "The monotonicity of the ratio between generalized logarithmic means," Journal of Mathematical Analysis and Applications, vol. 345, no. 1, pp. 86-89, 2008.

[7] B.-N. Guo and F. Qi, "A simple proof of logarithmic convexity of extended mean values," Numerical Algorithms, vol. 52, no. 1, pp. 89-92, 2009.

[8] Y.-M. Chu and W.-F. Xia, "Inequalities for generalized logarithmic means," Journal of Inequalities and Applications, vol. 2009, Article ID 763252, 7 pages, 2009.

[9] M.-Y. Shi, Y.-M. Chu, and Y.-P. Jiang, "Optimal inequalities among various means of two arguments," Abstract and Applied Analysis, vol. 2009, Article ID 694394, 10 pages, 2009.

[10] W.-F. Xia and Y.-M. Chu, "Optimal inequalities related to the logarithmic, identric, arithmetic and harmonic means," Revue d'Analyse Numérique et de Théorie de l'Approximation, vol. 39, no. 2, pp. 176 $183,2010$.

[11] Y.-M. Chu and W.-F. Xia, "Two optimal double inequalities between power mean and logarithmic mean," Computers E Mathematics with Applications, vol. 60, no. 1, pp. 83-89, 2010.

[12] W.-F. Xia, Y.-M. Chu, and G.-D. Wang, "The optimal upper and lower power mean bounds for a convex combination of the arithmetic and logarithmic means," Abstract and Applied Analysis, vol. 2010, Article ID 604804, 9 pages, 2010.

[13] B.-Y. Long and Y.-M. Chu, "Optimal inequalities for generalized logarithmic, arithmetic, and geometric means," Journal of Inequalities and Applications, vol. 2010, Article ID 806825, 10 pages, 2010. 
[14] Y.-M. Chu, S.-S. Wang, and C. Zong, "Optimal lower power mean bound for the convex combination of harmonic and logarithmic means," Abstract and Applied Analysis, vol. 2011, Article ID 520648, 9 pages, 2011.

[15] M.-Y. Shi, Y.-M. Chu, and Y.-P. Jiang, "Optimal inequalities related to the power, harmonic and identric means," Acta Mathematica Scientia A, vol. 31, no. 5, pp. 1377-1384, 2011 (Chinese).

[16] Y.-F. Qiu, M.-K. Wang, Y.-M. Chu, and G.-D. Wang, "Two sharp inequalities for Lehmer mean, identric mean and logarithmic mean," Journal of Mathematical Inequalities, vol. 5, no. 3, pp. 301-306, 2011.

[17] Y.-M. Chu, M.-K. Wang, and Z.-K. Wang, "A sharp double inequality between harmonic and identric means," Abstract and Applied Analysis, vol. 2011, Article ID 657935, 7 pages, 2011.

[18] Y.-M. Chu and M.-K. Wang, "Optimal inequalities between harmonic, geometric, logarithmic, and arithmetic-geometric means," Journal of Applied Mathematics, vol. 2011, Article ID 618929, 9 pages, 2011.

[19] Y.-M. Chu, S.-W. Hou, and W.-M. Gong, "Inequalities between logarithmic, harmonic, arithmetic and centroidal means," SIAM Journal on Mathematical Analysis, vol. 2, no. 2, pp. 1-5, 2011.

[20] H.-N. Hu, S.-S. Wang, and Y.-M. Chu, "Optimal upper power mean bound for the convex combiantion of harmonic and logarithmic means," Pacific Journal of Applied Mathematics, vol. 4, no. 1, pp. 35-44, 2011.

[21] Y.-F. Qiu, M.-K. Wang, and Y.-M. Chu, "The sharp combination bounds of arithmetic and logarithmic means for Seiffert's mean," International Journal of Pure and Applied Mathematics, vol. 72, no. 1, pp. 11-18, 2011.

[22] Y.-F. Qiu, M.-K. Wang, and Y.-M. Chu, "The optimal generalized Heronian mean bounds for the identric mean," International Journal of Pure and Applied Mathematics, vol. 72, no. 1, pp. 19-26, 2011.

[23] M.-K. Wang, Z.-K. Wang, and Y.-M. Chu, "An optimal double inequality between geometric and identric means," Applied Mathematics Letters, vol. 25, pp. 471-475, 2012.

[24] B.-N. Guo and F. Qi, "Inequalities for generalized weighted mean values of convex function," Mathematical Inequalities \& Applications, vol. 4, no. 2, pp. 195-202, 2001.

[25] A. O. Pittenger, "The logarithmic mean in $n$ variables," The American Mathematical Monthly, vol. 92, no. 2, pp. 99-104, 1985.

[26] P. Kahlig and J. Matkowski, "Functional equations involving the logarithmic mean," Zeitschrift für Angewandte Mathematik und Mechanik, vol. 76, no. 7, pp. 385-390, 1996

[27] G. Pólya and G. Szegö, Isoperimetric Inequalities in Mathematical Physics, Princeton University Press, Princeton, NJ, USA, 1951.

[28] E. B. Leach and M. C. Sholander, "Extended mean values. II," Journal of Mathematical Analysis and Applications, vol. 92, no. 1, pp. 207-223, 1983.

[29] J. Sándor, "A note on some inequalities for means," Archiv der Mathematik, vol. 56, no. 5, pp. 471-473, 1991.

[30] B. C. Carlson, “The logarithmic mean,” The American Mathematical Monthly, vol. 79, pp. 615-618, 1972.

[31] H. Alzer, "Ungleichungen für Mittelwerte," Archiv der Mathematik, vol. 47, no. 5, pp. 422-426, 1986.

[32] P. S. Bullen, D. S. Mitrinović, and P. M. Vasić, Means and Their Inequalities, vol. 31, D. Reidel, Dordrecht, The Netherlands, 1988.

[33] H. Alzer, “Ungleichungen fur $(e / a)^{a}(b / e)^{b}$," Elemente der Mathematik, vol. 40, no. 5, pp. 120-123, 1985.

[34] F. Burk, "Notes: the geometric, logarithmic, and arithmetic mean inequality," The American Mathematical Monthly, vol. 94, no. 6, pp. 527-528, 1987.

[35] T. P. Lin, "The power mean and the logarithmic mean," The American Mathematical Monthly, vol. 81, pp. 879-883, 1974.

[36] A. O. Pittenger, "Inequalities between arithmetic and logarithmic means," Univerzitet u Beogradu, vol. 678-715, pp. 15-18, 1980.

[37] A. O. Pittenger, "The symmetric, logarithmic and power means," Univerzitet u Beogradu, vol. 678-715, pp. 19-23, 1980.

[38] H. Alzer and S.-L. Qiu, "Inequalities for means in two variables," Archiv der Mathematik, vol. 80, no. 2, pp. 201-215, 2003.

[39] Y.-M. Chu and B.-Y. Long, "Best possible inequalities between generalized logarithmic mean and classical means," Abstract and Applied Analysis, vol. 2010, Article ID 303286, 13 pages, 2010. 


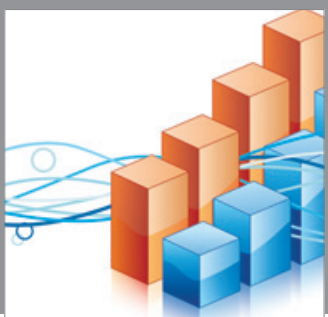

Advances in

Operations Research

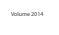

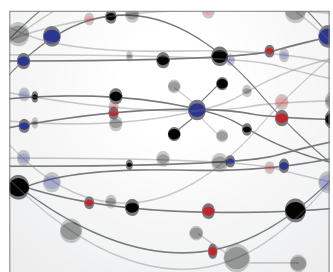

\section{The Scientific} World Journal


International Journal of

Mathematics and

Mathematical

Sciences
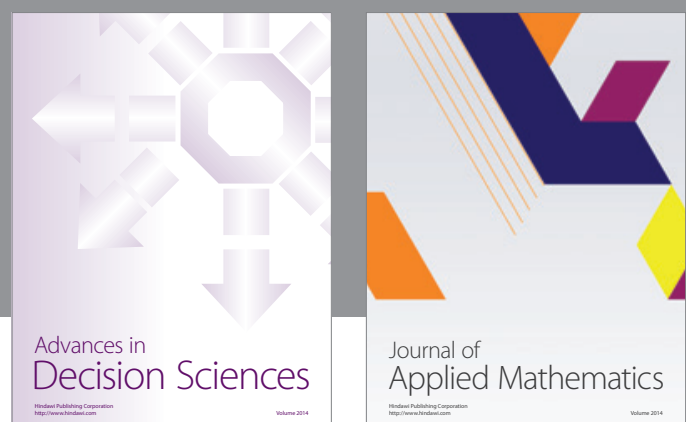

Journal of

Applied Mathematics
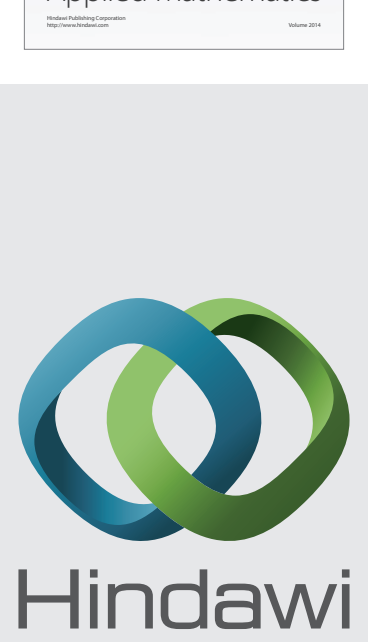

Submit your manuscripts at http://www.hindawi.com
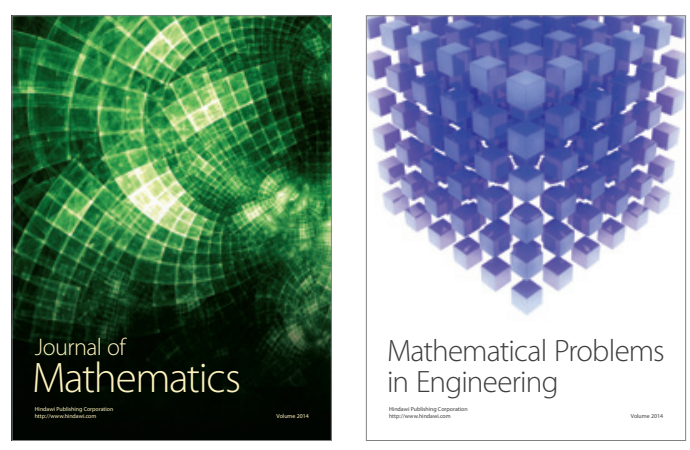

Mathematical Problems in Engineering
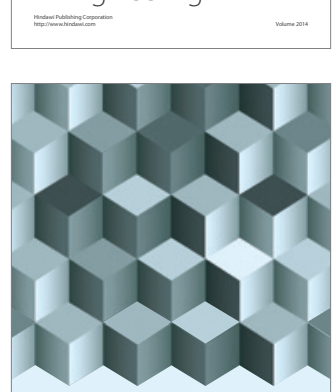

Journal of

Function Spaces
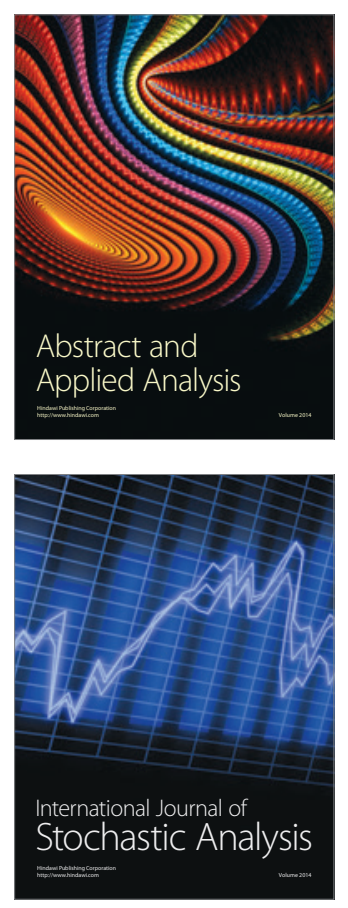

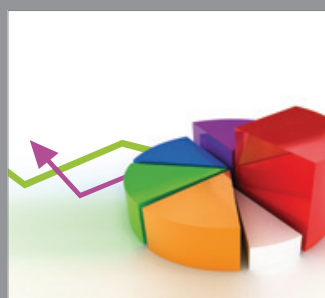

ournal of

Probability and Statistics

Promensencen
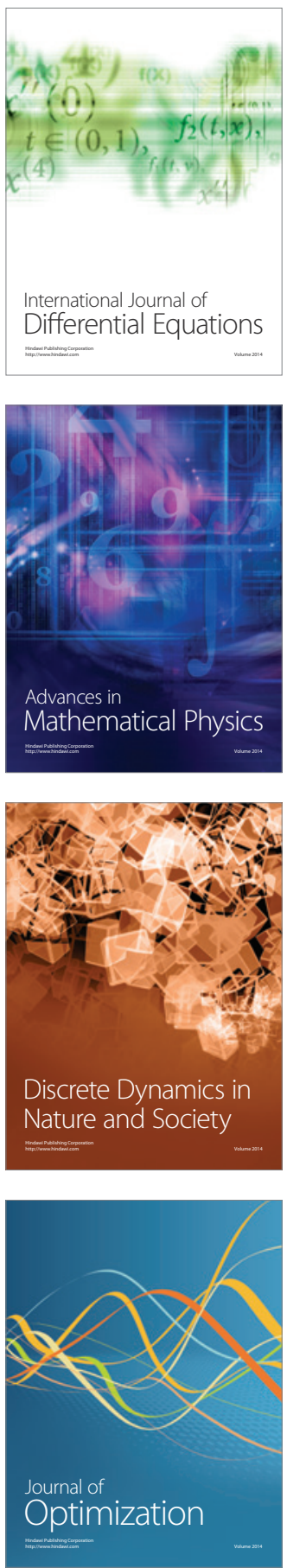